\title{
Ostomy Skin Barrier
}

National Cancer Institute

\section{Source}

National Cancer Institute. Ostomy Skin Barrier. NCI Thesaurus. Code C127126.

A device designed to adhere to the peristomal skin to protect it from the output of the stoma. It may be incorporated into a system for attaching an ostomy pouch. 